\title{
A6 HUMORAL IMMUNE RESPONSE AGAINST FIBRINOGEN EPITOPES CITRULLINATED IN VIVO IN RHEUMATOID ARTHRITIS SYNOVIAL TISSUE DETECTED BY AUTOANTIBODY MULTIPLEXING
}

Johan Rönnelid, ${ }^{1}{ }^{2}$ Elena Ossipova, ${ }^{1}$ Linda Mathsson, ${ }^{2}$ Anca Catrina, ${ }^{1}$ Per Matsson, ${ }^{3}$ Lena Israelsson, ${ }^{1}$ Vivianne Malmström, 'Lars Klareskog, ${ }^{1}$ Per-Johan Jakobsson ${ }^{1}$ 'Unit of Rheumatology, Department of Medicine, Karolinska Institutet, Stockholm, Sweden; ${ }^{2}$ Unit of Clinical Immunology, Uppsala University, Uppsala, Sweden; ${ }^{3}$ Phadia AB, Uppsala, Sweden

\subsection{6/ard.2010.149096.6}

Background The anticitrullinated protein/peptide antibody (ACPA) response in rheumatoid arthritis (RA) might be driven by key autoantigens citrullinated in the target organ. We have recently by using high definition mass spectrometry defined fibrinogen sequences which are citrullinated in RA synovial tissue. $^{1}$

Objective To use a newly developed multiplexing assay to investigate the humoral immune response against such in vivo citrullinated fibrinogen epitopes.

Methods The Phadia ImmunoCAP ISAC chip was used to bind four synthetic fibrinogen peptides containing citrulline as shown in vivo corresponding to the $\alpha$ chain positions 573 and 591, the $\beta$ chain positions 72 and 74 , as well as their arginine-containing control peptides. 848 RA patients and 150 controls from the Epidemiological Investigations in Rheumatoid Arthritis cohort were investigated. Peptide-specific ELISA tests were developed for validation of the microarray in a smaller group of RA sera $(n=123)$ and controls $(n=20)$.

Results Using the 98th percentile among the controls, $13.3 \%$ $(113 / 848)$ of the RA patients had antibodies against the citrullinated 573 peptide. There was a substantial reactivity also to the arginine control peptide both in RA patients and controls, not generally observed for other arginine-containing control peptides. After subtraction of the arginine response, the net immune response discriminated better between patients and controls, and using the 98th percentile for the net citrulline response among $\mathrm{HC}, 35.5 \%$ (301/848) of the RA patients were autoantibody positive. The area under the receiver operator characteristics (ROC) curve was 0.610 for uncorrected values, but after subtraction of the readout for arginine controls the AUC increased to 0.800 . The microarray showed good correlation to autoantibody levels measured with ELISA ( $r=0.83)$. The four peptides showed considerably divergent responses in ROC curve analyses both before and after correction for arginine control peptides, and the remaining three citrullinated peptides are currently validated.

Conclusion This study proves a substantial autoantibody reactivity against fibrinogen epitopes citrullinated in vivo in RA synovial tissue. Autoantibody multiplexing can be used for the screening humoral reactivities against large numbers of potential autoantigenic epitopes, for example, those found citrullinated in vivo. This technique also allows easy correction for autoantibody reactivity against the arginine-containing peptide backbone normally not performed with ACPA ELISA tests. Concerning the fibrinogen 573 peptide, such correction increased the diagnostic performance of autoantibody measurement considerably.

\section{REFERENCES}

1. Hermansson M, Artemenko K, Ossipova E, et al. MS analysis of rheumatoid arthritic synovial tissue identifies specific citrullination sites on fibrinogen. Proteomics Clin Appl, 2010;4(5):511-8. 